\title{
Hand Gesture Detection Using Segmentation
}

\author{
Nishu Sethi ${ }^{1}$, Shivangi Kaushal ${ }^{2}$, and Neha Bhateja ${ }^{3}$ \\ ${ }^{1,2,3}$ Assistant Professor, Department of Computer Science \& Engineering, Amity University, Gurugram, Haryana, India
}

Correspondence should be addressed to Nishu Sethi; sethinishu@gmail.com

Copyright ( 2021 Made Nishu Sethi et al. This is an open access article distributed under the Creative Commons Attribution License, which permits unrestricted use, distribution, and reproduction in any medium, provided the original work is properly cited.

\begin{abstract}
Hand gesture detection is a project which recognizes the gesture of hands and detect accordingly. Hand Gesture recognition is an important technique for creating user-friendly interfaces. Hand gesture is recognized by robots, for example, can take human commands, and those who are deaf or who cannot speak, can recognize the sign language for communication. Hand gesture recognition in video games could help by allowing players to use gestures to interact with the game rather than a controller. Moreover, to account for the infinite number of possible hand positions in three dimensions, such an algorithm must be more robust. It must also be capable of working with video rather than static images.
\end{abstract}

KEYWORDS- Gesture, human-computer, segmentation, user interface.

\section{INTRODUCTION}

A Hand Gesture Recognition System recognizes the orientations and shapes which depends on how our system is implemented to perform some tasks. A gesture is a type of nonverbal communication. An individual can make multiple signs at the same time. For example, humans perceive human gestures through visualization and computers require a camera, A topic of particular interest to processor vision examiners, such as carrying out an act based on a person's gestures. Most people's daily lives revolve around human-computer interaction. The old human-computer interaction approach [1], which includes everything from the inventive keyboard to today's joystick, wireless input devices and mouse, greatly simplifies interaction of computer with people, making it easier to operate computers and enhance efficiency of work. Consequently, due to reliance on added hardware input devices, the demands of human-computer interaction cannot be fully met by interaction mode. Hand gestures[8] is defined as a collection of signs or activities made by combining arms or hands; they are always capable of expressing the intention of the signer, and thus can serve as natural means of communication among machine and human. The research is critical for the growth of new human-centered human-computer interactions. This paper examined the existing state of research and applications of the recognition. With the goal of summarizing the frequently used hand gesture recognition approaches, analyzing the strengths, weaknesses, and listing interesting glitches in existing hand gesture recognition study.

\section{A. Architecture of Gesture Recognition System}

We plan to use a webcam to implement a system that recognizes gesture input. The application is configured to run in background and the user can perform other tasks, which is extremely helpful for a hands-free approach. This project has an extensive range of potential applications, most remarkably the "Sixth Sense project", which completely revolutionizes the digital world. This code could be stretched to include the movement of mouse and the 3-D still gestures [2]. For improving the effectiveness of the gesture recognition process, better gesture interpretation and recognition can be improved, and newer gestures can be incorporated for increased functionality [3,7]. The user interface for calculating, examining gestures, and running the program, could greatly be enhanced by using an interactive GUI rather than terminal commands.

\section{LITERATURE REVIEW}

To recognize hand gestures using non-geometric features, Hasan used a multivariate Gaussian distribution [13,14]. Two methods are used to segment the input hand image: skin colour-based segmentation using the cluster-based threshold techniques and HSV colour model. Specific procedures are implemented to capture the hand's outline to extract the hand feature, the improved movement. The analysis algorithm calculates the trend and slope of object (hand) by determining the direction of hand movement, and it is used to discover a connection among statistical parameters (variance and covariance) in data. Using a neural network [10] algorithm, Kulkarni recognizes static postures in American Sign Language. The source image is converted to the HSV colour model, which is further resized to $80 \times 64$, and image pre-processing operations are performed to extract features from uniform background and hand using the histogram technique and the Hough algorithm [11]. Feeding back for gesture classification, three-layer neural networks are used. They achieved 92.78 percent recognition rate with 8 samples for 26 sign language characters in, 3 testing samples and 5 training samples.Hasan used scaled normalization to recognize gestures on brightness factor matching [14]. Source image is sectioned using threshold technique with black background. Any segmented image is normalized, and image's centre mass is determined, and the coordinates are shifted to match the centroid of the hand object at the $\mathrm{X}$ and $\mathrm{Y}$ axis origins. Wysoski et al. [5] used a boundary histogram to present rotation invariant postures. The input image was captured using a camera, a filter for skin color detection was applied, and a clustering process was used to use an ordinary contour-tracking algorithm for finding the 
boundary of every cluster in the clustered image. Grids were drawn on the image, and edges were normalized.

\section{CHALLENGES}

Despite significant progress and high recognition rates in various areas, the study still faces numerous challenges including transition model between gestures, extraction of invariant features, automatic segmentation of recognition units, minimal sign language recognition units [6], auxiliary information, recognition approach with scalability about vocabularies, mixed gestures recognition, signer independent and so on. The current trend is static gesture recognition based on vision and is characterized by two technical challenges defined as:

\section{A. Target Detection Difficulties}

The goal of target detection is to extract the object of interest from an image stream by capturing the target. Due to the variety of background and unforeseen environment factors [9], it is necessary in vision-based hand gesture recognition methods to divide the human hand area and other background areas in the image.

\section{B. Difficulties in Recognizing Targets}

The purpose of hand gesture recognition is to describe the top implications of hand's changing and posture process [12]. The vital skill is the extraction of geometric invariant features based on hand gesture characteristics.

\section{PROPOSED SYSTEM}

Dynamic and Static recognition are the types of hand gesture recognition. Static technique recognizes shape of hand and reads the meaning of the hand expression. The acknowledgement of a hand signal trajectory in space and performing the operation on the trajectory constraints obtained is referred to as dynamic hand gesture recognition. A hand gesture is used to flip down and up, start, and pause the courseware on a projection. To begin, the background subtraction method is used to detect hand, and resulting binary image is transformed.

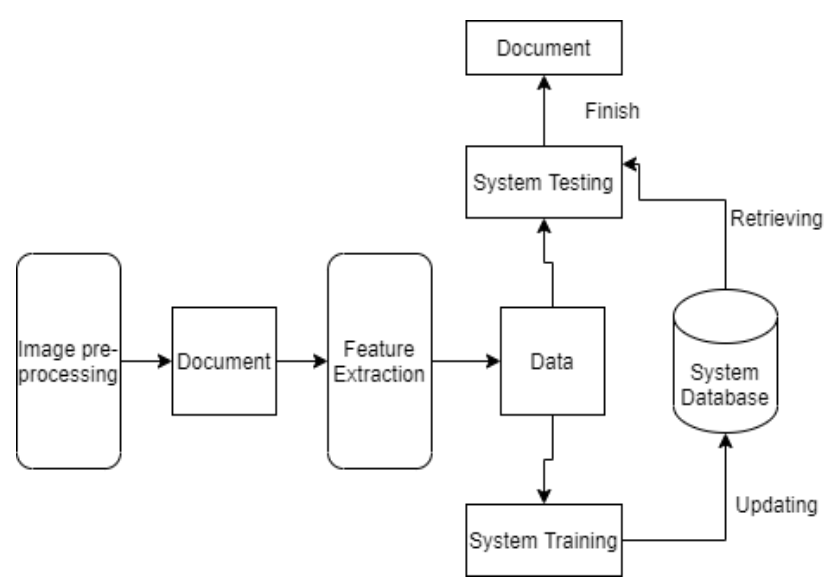

Fig. 1: Workflow of Hand Recognition System [4]

\section{A. Results}

Following are the results obtained by classifying the number of figures.

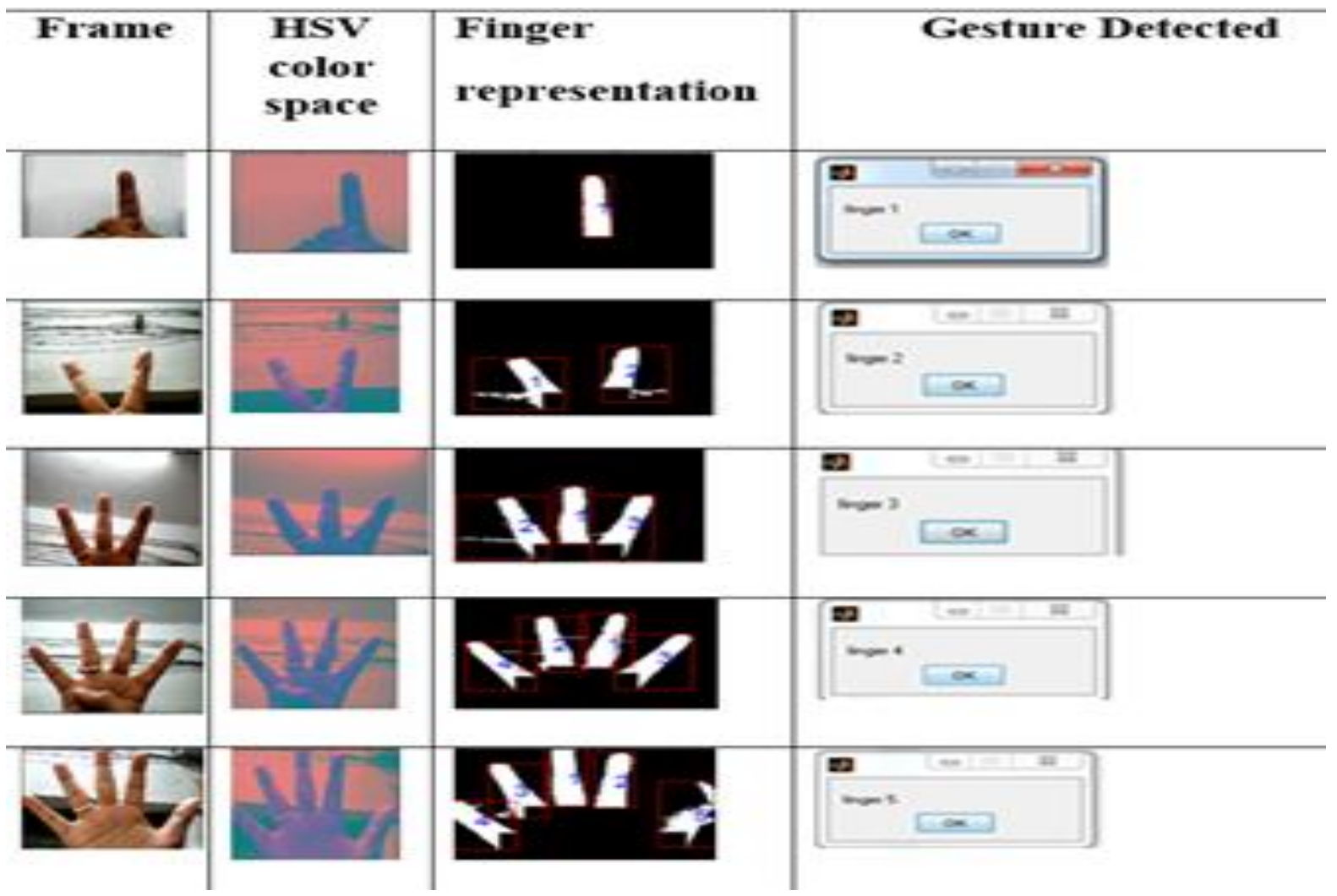

Fig. 2: Screenshot of hand gesture of numeral fingers 
Here in this paper, the images are manually captured and then processing of those images is done which shows the static hand gesture. Another thing that should be noticed here is that the counting of the fingers is also done for hand gesture representation.

\section{CONCLUSION}

This paper introduces a new method for recognizing hand gestures. The background subtraction method detects the hand region from background. Then, the palm and fingers are fragmented. The fingers in hand image are discovered and recognized using segmentation. A straightforward rule classifier is used to recognize hand gestures.

The performance of projected technique depends on the outcome of hand detection. If the objects are moving with skin-like colors, they exist because of hand detection and degrade hand gesture recognition performance. Machine learning algorithms, on the other hand, can tell the difference between hand and background.

\section{CONFLICTS OF INTEREST}

The authors declare that they have no conflicts of interest.

\section{REFERENCES}

[1] Pavlovic, V. I., Sharma, R., \& Huang, T. S. (1997). Visual interpretation of hand gestures for human-computer interaction: a review. IEEE Transactions on Pattern Analysis and Machine Intelligence, 19(7), 677695. doi:10.1109/34.598226.

[2] Mokhtar M. Hasan, Pramoud K. Misra, (2011). "Brightness Factor Matching for Gesture Recognition System Using Scaled Normalization", International Journal of Computer Science \& Information Technology (IJCSIT), Vol. 3(2).

[3] Xingyan Li. (2003). "Gesture Recognition Based on Fuzzy CMeans Clustering Algorithm", Department of Computer Science. The University of Tennessee Knoxville.

[4] S. Mitra, and T. Acharya. (2007). "Gesture Recognition: A Survey" IEEE Transactions on systems, Man and Cybernetics, Part C: Applications and reviews, vol. 37 (3), pp. 311- 324, doi:10.1109/TSMCC.2007.893280.

[5] Simei G. Wysoski, Marcus V. Lamar, Susumu Kuroyanagi, Akira Iwata, (2002). "A RotationInvariant Approach on Static-Gesture Recognition Using Boundary Histograms and Neural Networks" International Journal of Artificial Intelligence \& Applications (IJAIA), Vol.3, No.4, July 2012, 173, IEEE Proceedings of the 9th International Conference on Neural Information Processing, Singapura.

[6] Joseph J. LaViola Jr., (1999). "A Survey of Hand Posture and Gesture Recognition Techniques and Technology", Master Thesis, Science and Technology Center for Computer Graphics and Scientific Visualization, USA.

[7] Rafiqul Z. Khan, Noor A. Ibraheem, (2012). "Survey on Gesture Recognition for Hand Image Postures", International Journal of Computer and Information Science, Vol. 5(3), Doi: $10.5539 /$ cis.v5n3p110.

[8] Thomas B. Moeslund and Erik Granum, (2001). "A Survey of Computer Vision-Based Human Motion Capture," Elsevier, Computer Vision and Image Understanding, Vol. 81, pp. 231268.

[9] N. Ibraheem, M. Hasan, R. Khan, P. Mishra, (2012). "Comparative study of skin color based segmentation techniques", Aligarh Muslim University, A.M.U., Aligarh, India.

[10] E. Stergiopoulou, N. Papamarkos. (2009). "Hand gesture recognition using a neural network shape fitting technique," Elsevier Engineering Applications of Artificial Intelligence, vol.

22(8),

doi:10.1016/j.engappai.2009.03.008

pp.1141-1158,

[11] M. M. Hasan, P. K. Mishra, (2011). "HSV Brightness Factor Matching for Gesture Recognition System", International Journal of Image Processing (IJIP), Vol. 4(5).

[12] Malima, A., Özgür, E., Çetin, M. (2006). "A Fast Algorithm for Vision-Based Hand Gesture Recognition for Robot Control", IEEE 14th conference on Signal Processing and Communications Applications, pp. 1-4. doi: 10.1109/SIU.2006.1659822.

[13] Mokhar M. Hasan, Pramod K. Mishra, (2012) "Features Fitting using Multivariate Gaussian Distribution for Hand Gesture Recognition", International Journal of Computer Science \& Emerging Technologies IJCSET, Vol. 3(2).

[14] Mokhar M. Hasan, Pramod K. Mishra, (2012). "Robust Gesture Recognition Using Gaussian Distribution for Features Fitting', International Journal of Machine Learning and Computing, Vol.2(3)

\section{ABOUT THE AUTHORS}

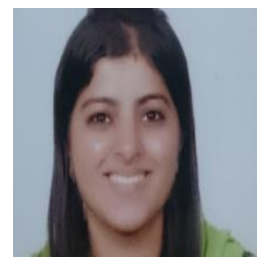

Ms Nishu Sethi is currently designated as Assistant Professor in CSE Department at Amity University Haryana. She has published more than 15 research papers. She is an active Member and Reviewer of more than 8 reputed Journals.

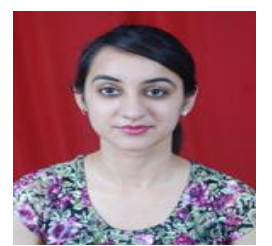

Ms Shivangi Kaushal is currently designated as Assistant Professor in CSE at Amity University Haryana. She has published more than 15 research papers. She is an active Member and Reviewer of more than 7 reputed Journals.

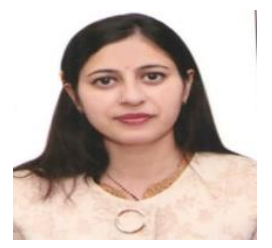

Ms Neha Bhateja is currently designated as Assistant Professor in CSE at Amity University Haryana. She has published more than 18 research papers. She is an active Member and Reviewer of more than 7 reputed Journals. 